\title{
Pulmonary Mucormycosis with Diabetes Mellitus: A Case Report
}

\author{
SHIKHA JAIN', BHARAT SHARMA²
}

Mucormycosis is an uncommon but life threatening fungal infection that generally occurs mostly in immunocompromised patients. Patients do not respond to the antibiotics and so the condition may prove to be fatal if not timely detected. In this report, we summarize a case of pulmonary mucormycosis in a patient with diabetes mellitus who was cured using Amphotericin B.

KEYWORDS: Pulmonary Mucormycosis, Rhizopus, Diabetes Mellitus

\section{INTRODUCTION}

Besides the infectious diseases of bacterial origin, fungal infections are also commonly observed more often in patients in the intensive care unit. The most common fungus causing infection is the Candida species, especially among the immunocompromised patients. The most common cause of pulmonary infections is still Aspergillus species, but there has been a rise in infections due to other fungii, namely Mucor and Rhizopus. The infection caused by these fungal species is known as Mucormycosis (previously called zygomycosis). These patients present with various risk factors like a central venous catheter, receiving parenteral nutrition, broad-spectrum antibiotics or it may be a patient who has undergone surgical procedure or hemodialysis.

\section{CASE REPORT}

A 69 year old male with type 2 diabetes mellitus and having a medical history of coronary artery disease presented with intermittent fever, productive cough, breath shortness and loss of appetite for one month. Patient was evaluated on the lines of pneumonia. Growth of Staphylococcus haemolyticus was seen in the bacterial culture of the sputum. Patient was already on high end antibiotics at the time of admission to hospital, but was not responding to them.

\section{Radiological findings: A high Resolution Computed Tomography scan revealed fibroatelectatic lesions in the anterior segment of both upper lobe and basal segment of left lower lobe along with parenchymal infilterative lesions in the apico-posterior and anterior segment of left}

upper and posterior basal segment of right lower lobe.

Laboratory findings: Patient was evaluated for tuberculosis and fungal infection. Report for Mycobacterium tuberculosis was negative on Genexpert and mantoux test was also negative. The sputum specimen was then received in the laboratory for examination of fungus by potassium hydroxide preparation ( $\mathrm{KOH}$ preparation) and fungal culture.

Potassium hydroxide preparation of sputum specimen showed many broad, aseptate fungal hyphae with right-angle branching. Fungal culture was carried out and the colonies obtained on culture were dense and had a white hairy appearance. Staining was done using lactophenol cotton blue mount preparation which showed nodal rhizoids, pyriform to ellipsoidal-shaped columella, short subglobose to slightly angular sporangiospores, missing observable striations indicative of Rhizopus species. Identification of the fungal isolate was confirmed on Vitek 2 (automated culture) as Rhizopus species and patient was put on antifungal therapy (Amphotericin B) as per the sensitivity testing report to which he responded in a very well manner.

\section{DISCUSSION}

Mucormycosis are a group of invasive, medically significant infections, which are caused by filamentous fungi of the order, Mucorales of the Mucoraceae Family. The risk factors behind these 
infections are ailments like diabetes mellitus, hematological malignant disorders and a prolonged corticosteroid or immunosuppressive medication. Following Aspergillus and Candida species, the third leading cause of invasive fungal infections are members of the order mucorales. The most frequently isolated species are Rhizopus oryzae, Rhizopus microsporeus and Absidia corymbifera. ${ }^{1}$

Among different categories of zygomycosis, the most commonly occurring is the rhino-orbitalcerebral type which is succeeded by Pulmonary zygomycosis with common presenting symptoms like productive cough, increase in body temperature and pleuritic chest pain and it has seldom been reported without any predisposing determinant. ${ }^{2}$ Along with non specific symptoms, chest radiographs show involvement of the upper part of the chest in nearly half of the patients. ${ }^{3}$

Without prompt therapeutic management, invasive zygomycosis invariably proves to be lifethreatening or fatal. Aggressive surgery, suitable and well judged medical therapy, and control of predisposing elements are of prime significance in the treatment of infected cases. ${ }^{4}$ Amphotericin B, in lipid preparations is top of the line drug of choice for most of the cases of zygomycosis and most of the patients respond well to the therapy.

\section{CONCLUSION}

Critical patients not responding to even high end antibiotics must be suspected and detected for fungal infections. It is essential to make timely diagnosis and start antifungal treatment so that systemic fungal infections which otherwise may prove to be fatal, can be cured.

\section{REFERENCES}

1. Shinde RV, Karande GS, Mohite ST, Patil SR. Rhino-orbital mucormycosis in diabetes mellitus. J Clin Diagn Res 2013; 7(6): 1145-7.

2. Tedder M, Spratt JA, Anstadt MP, Hedge SS, Tedder SD, Lowe JE. Pulmonary mucormycosis: results of medical and surgical therapy. Ann Thorac Surg 1994; 57(4):1044-50.

3. Lee FY, Mossad SB, Adal KA. Pulmonary mucormycosis: the last 30 years. Arch Intern Med. 1999;159(12):1301-9.

4. Chakrabarti A, Das A, Sharma A, Panda N, Das $\mathrm{S}$ et al. Ten years' experience in zygomycosis at a tertiary care centre in India. J Infect 2001;42:261-6.

Source of support: Nil, Conflict of interest: None declared

Cite this article as:

Jain S, Sharma B. Pulmonary Mucormycosis with Diabetes Mellitus: A Case

Report. Int Healthcare Res J 2018;2(2):33-34. doi: 10.26440/IHRJ/02_02/164

\section{AUTHOR AFFILIATIONS:}

1. Consultant Microbiologist, Max Hospital, Mohali, Punjab, India

2. MBBS, Senior Lecturer, General Medicine, Shri Sukhmani Dental College and Hospital, Dera Bassi, Mohali, Punjab, India

\section{Corresponding Author:}

Dr. Shikha Jain

Consultant Microbiologist

Max Hospital, Mohali

Punjab, India
For article enquiry/author contact details, e-mail at: manuscriptenquiry.ihrj@gmail.com 